\title{
Cinética de Vulcanização de Composições de Borracha Natural com Incorporação de Cinza de Casca de Arroz
}

\author{
Helson M. da Costa, Leila L. Y. Visconte, Regina C. R. Nunes \\ IMA, UFRJ \\ Cristina R. G. Furtado \\ Grupo de Polímeros, IQ, UERJ
}

\begin{abstract}
Resumo: Cinza da casca de arroz foi incorporada em borracha natural (NR) utilizando-se um misturador de cilindros. O sistema de vulcanização convencional (CV) foi escolhido e os estudos sobre a cura das composições foram conduzidos em um Curômetro TI-100. As curvas de torque foram obtidas em 150,160, 170 e $180{ }^{\circ} \mathrm{C}$. A velocidade global e a energia de ativação aparente para o processo de vulcanização foram calculadas para cada composição, assumindo que a vulcanização segue uma cinética de primeira ordem. Para fins de comparação, duas cargas comerciais, sílica precipitada (Zeosil-175) e negro de fumo (N762), foram também usadas. Foi observado que a adição da cinza da casca de arroz às composições de $\mathrm{NR}$, em comparação às outras cargas utilizadas, aumentou a velocidade de reticulação e diminuiu a energia de ativação aparente de modo mais marcante.
\end{abstract}

Palavras-chave: Borracha natural, vulcanização, cinética, cinza da casca de arroz.

\section{Vulcanization Kinetics of Natural Rubber Filled with Rice Husk Ash}

Abstract: Rice husk ash was incorporated into natural rubber (NR) using a laboratory size two-roll mill. A conventional vulcanization system $(\mathrm{CV})$ was chosen and cure studies were carried out on a TI-100 Curometer. The torque curves were obtained at $150,160,170$, and $180^{\circ} \mathrm{C}$. The overall rate and the apparent activation energy for the vulcanization process were calculated for each compound assuming that vulcanization follows first-order kinetics. For the purpose of comparison, two commercial fillers, precipitated silica (Zeosil-175) and carbon black (N762), were also used. In comparison to the other fillers used, the addition of rice husk ash to NR compounds increased the crosslinking rate and lowered the apparent activation energy in a more marked way.

Keywords: Natural rubber, vulcanization, kinetics, rice husk ash.

\section{Introdução}

A produção de arroz, uma das maiores culturas agrícolas do mundo, gera grande quantidade de resíduos, dentre eles as cascas e palhas. O descarte das cascas é um problema particularmente sério, e requer especial atenção devido à grande quantidade acumulada nas proximidades dos moinhos de arroz. As cascas quando queimadas a céu aberto produzem uma cinza que apresenta bom potencial como carga para elastômeros, pois sua composição química consiste predominantemente de sílica ${ }^{[1,2]}$.

A sílica é, juntamente com o negro de fumo, a carga de reforço mais usada em polímeros, em particular em composições elastoméricas. Embora o negro de fumo seja inegavelmente a carga mais amplamente usada, o crescente conhecimento que se vem adquirindo sobre o fenômeno de reforço tem levado ao emprego cada vez maior da sílica, isoladamente ou em combinação com outros materiais, devido a algumas características interessantes provocadas pela presença dessa carga. O caráter ácido da sílica, no entanto, provoca retardamento da reação de vulcanização se nenhuma substância que possa corrigir o $\mathrm{pH}$ foi adicionada. Outro grande problema com o uso da sílica é o fato de ser uma carga relativamente cara e, por conseguinte, produzir vulcanizados onerosos. Portanto, a busca por materiais que possam substituir total ou parcialmente a sílica, sem prejuízo das propriedades, torna-se importante. A cinza da casca de arroz apresenta-se, então, como um potencial substituto bastante promissor ${ }^{[3]}$.

Dependendo de como a cinza é gerada, duas variedades são produzidas, as quais diferem principalmente no percentual em sílica. A cinza branca origina-se a temperaturas maiores e é quase totalmente composta por sílica, enquanto a cinza preta, formada a temperaturas mais baixas, contém, além da sílica, uma boa quantidade de material orgânico.

Materiais para serem usados como carga em composições elastoméricas devem diminuir o custo final do produto, sem prejuízo de suas propriedades ou de suas características de processamento. Além disso, não devem interferir no processo de vulcanização que é a etapa crucial na fabricação de arte-

Autor para correspondência: Regina C. R. Nunes, IMA, UFRJ, Caixa Postal 68525, CEP: 21945-970, Rio de Janeiro, RJ. E-mail: rcnunes@ima.ufrj.br 
fatos de borracha. Portanto, um bom entendimento dos parâmetros envolvidos na vulcanização é fundamental para o processamento adequado de composições elastoméricas.

Técnicas comuns utilizadas no estudo da vulcanização de borrachas incluem calorimetria de varredura diferencial (DSC), análise química e reometria de disco oscilatório (curômetro). A técnica de DSC é baseada na hipótese de que o calor de reação está relacionado unicamente com a reação de formação de ligações cruzadas e é proporcional à extensão da reação, o que em sistemas complexos, muitas vezes é questionável. A análise química envolve um número considerável de reações e um consumo de tempo excessivo. $\mathrm{O}$ estudo por meio do curômetro é baseado no fato de que a densidade de ligações cruzadas é proporcional ao torque registrado durante a vulcanização das composições de borracha ${ }^{[4]}$.

De acordo com Chough e Chang ${ }^{[4]}$, a energia de ativação aparente $\left(E_{a}\right)$ para composições de borracha pode ser obtida a partir de dados do reômetro. Assumindo-se que a vulcanização segue uma cinética de primeira ordem, a expressão cinética dada pela equação (1) para a vulcanização, pode ser definida em termos do torque medido:

$$
\ln \left(\frac{\mathrm{M}_{\mathrm{h}}-\mathrm{M}_{\mathrm{l}}}{\mathrm{M}_{\mathrm{h}}-\mathrm{M}_{\mathrm{t}}}\right)=\mathrm{kt}
$$

onde $M_{t}$ é o torque em um tempo t; $M_{l}$ e $M_{h}$ representam o torque mínimo e máximo, respectivamente; e $k$ é a constante de velocidade para a vulcanização. Valores de $M_{t}$ para $25 \mathrm{e}$ $45 \%$ do torque máximo foram escolhidos para estimar a constante de velocidade. A combinação da equação de Arrhenius com a equação (1) gera a equação (2), conveniente para o cálculo de $E_{a}$, a partir do uso de isotermas obtidas no reômetro:

$$
\frac{E_{a}}{R}=\ln \left[\frac{\ln \left(\frac{\left(t_{45 \%}-t_{25 \%}\right)_{\beta}}{\left(t_{45 \%}-t_{25 \%}\right)_{\alpha}}\right)}{\frac{1}{T_{\beta}}-\frac{1}{T_{\alpha}}}\right]
$$

onde $t_{25 \%}$ e $t_{45 \%}$ são os tempos correspondentes a $25 \%$ e $45 \%$ do valor de $M_{h}$, respectivamente; e $T_{\alpha}$ e $T_{\beta}$ são as temperaturas das isotermas ${ }^{[4]}$.

Neste trabalho, o efeito da cinza da casca de arroz sobre a energia de ativação aparente $\left(E_{a}\right)$ para a vulcanização de composições de borracha natural foi estudado. Duas variedades de cinza foram usadas: cinza preta (CP), com teor de $\mathrm{SiO}_{2}$ em torno de $70 \%$, e a cinza branca (CB), cujo teor de $\mathrm{SiO}_{2}$ está em torno de $97 \%$. Cargas comerciais de negro de fumo (N762) e sílica (Zeosil-175) foram utilizadas para fins de comparação. A substituição parcial das cargas comerciais pelas cinzas $\mathrm{CP}$ e CB também foi conduzida e o efeito sobre a vulcanização avaliado.

\section{Experimental}

A formulação utilizada foi (em phr): borracha natural, 100; ácido esteárico, 2,5; óxido de zinco, 3,5; antioxidante, 2,0; cargas 0-50, acelerador (CBS), 0,8; enxofre, 2,5. Cada uma das
Tabela 1. Propriedades das diferentes cargas

\begin{tabular}{lcccc}
\hline \multicolumn{1}{c}{ Propriedade } & CP & CB & Sílica & $\begin{array}{c}\text { Negro } \\
\text { de fumo }\end{array}$ \\
\hline Tamanho médio de partícula $(\mu \mathrm{m})$ & 2,5 & 2,2 & 0,018 & 0,054 \\
Área específica $\left(\mathrm{m}^{2} / \mathrm{g}\right)$ & 109 & 17 & 185 & 30 \\
Área dos microporos $\left(\mathrm{m}^{2} / \mathrm{g}\right)$ & 88,2 & 1,8 & 48,3 & 3,7 \\
Área relativa a microporos $(\%)$ & 80,1 & 22,6 & 31,0 & 12,5 \\
Densidade $\left(\mathrm{g} / \mathrm{cm}^{3}\right)$ & 1,9 & 2,0 & 2,0 & 1,9 \\
$\mathrm{pH}$ & 9,5 & 9,4 & 6,5 & 6,4 \\
\hline
\end{tabular}

cinzas foi moída por $5 \mathrm{~h}$ e peneirada em $325 \mathrm{mesh}$. As propriedades das diferentes cargas estão resumidas na Tabela 1 .

A caracterização química das cinzas foi realizada através de espectrometria de emissão atômica com fonte de plasma acoplado indutivamente, utilizando-se o equipamento da Thermo Jarrel Ash, modelo IRIS/AP, após digestão das amostras com tetraborato de lítio.

As diferentes composições foram obtidas por meio de um misturador de cilindros a $70{ }^{\circ} \mathrm{C}$, de acordo com a norma ASTM D-3182. Neste trabalho, as curvas de torque foram obtidas a 150,160, 170 e $180^{\circ} \mathrm{C}$ em um Curômetro TI-100, fabricado pela Tecnología Industrial, e a cinética de vulcanização foi avaliada mediante a análise em triplicatas para cada composição.

\section{Resultados e Discussão}

A rota geral do processo de vulcanização é descrita pelo Esquema 1. Primeiro, um complexo ativo do acelerador é formado através de uma interação preliminar entre o acelerador e o ativador, na presença de zinco solúvel. Esse complexo pode reagir com o enxofre molecular, mediante a abertura do anel de $\mathrm{S}_{8}$, para formar um agente sulfurante ${ }^{[5-8]}$. A existência desses complexos foi detectada quimicamente ${ }^{[6,7]} \mathrm{e} \mathrm{tam}$ bém pela combinação das análises de NMR/HPLC ${ }^{[8]}$.

Posteriormente, o agente sulfurante pode reagir com as cadeias de borracha para formar um precursor de ligações cruzadas. O precursor foi detectado por evidências experimentais $^{[9-11]}$ na forma de um polissulfeto, ligado a um fragmento da molécula do acelerador, e está presente como grupo pendente na cadeia principal de borracha. $\mathrm{O}$ precursor, subseqüentemente, leva à formação de ligações cruzadas polissulfídicas.

Neste meio tempo, a diminuição da eficiência na formação de ligações cruzadas pode ocorrer devido a reações laterais, como decomposição ou dessulfurização dos precursores ${ }^{[1-14]}$. Por causa destas reações laterais, a formação de sulfetos cíclicos, dienos conjugados, trienos, $\mathrm{ZnS}$, e grupos monossulfídicos pendentes pode ser observada. Estas espécies não são capazes de contribuir para a geração de ligações cruzadas. Foi observado que a atividade, a concentração do complexo zinco-acelerador ${ }^{[13-15]}$ e a temperatura são as principais variáveis de controle para as possíveis reações laterais citadas. 


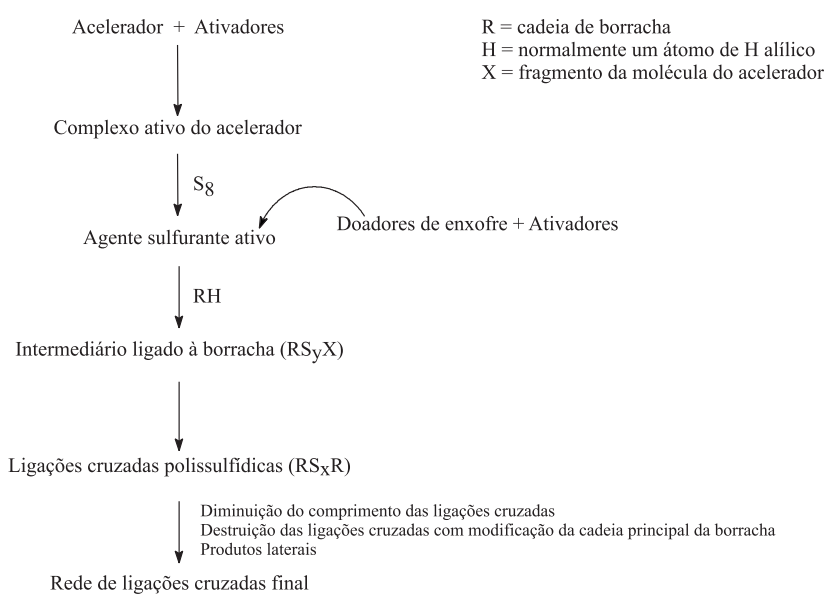

Esquema 1. Rota geral para o processo de vulcanização com acelerador e enxofre ${ }^{[9]}$

Finalmente, a rede de ligações cruzadas inicialmente formada sofre maturação e, durante esse processo, a dessulfurização (diminuição do tamanho das ligações cruzadas, eventualmente levando a ligações monossulfídicas) e/ou a decomposição das ligações cruzadas polissulfídicas, ocorrem ${ }^{[15-17]}$.

Os valores para $E_{a}$ das diferentes composições, calculados pela equação 2, estão ilustrados na Figura 1. Os resultados obtidos permitem concluir que:

a) Os altos valores de $E_{a}$, que representam baixas velocidades de cura para as composições com sílica podem ser atribuídos à interação sílica-ativador. A sílica reage com estearato de zinco (resultante da reação entre o $\mathrm{ZnO}$ e o ácido esteárico presentes na formulação) reduzindo a disponibilidade do estearato no processo de cura com o enxofre (Figura 2). Como nenhum ingrediente adicional (aminas ou glicóis), necessário para a correção do efeito adverso, foi considerado neste

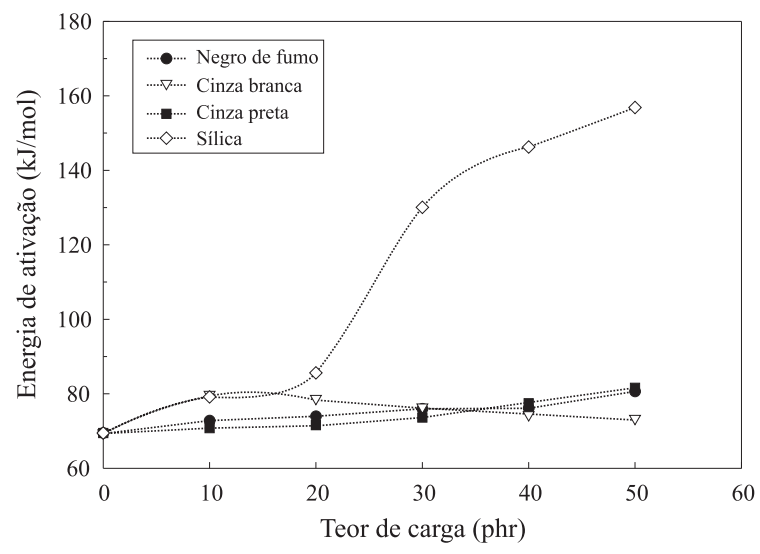

Figura 1. Variação da energia de ativação aparente para a vulcanização das composições de borracha natural contendo as cargas comerciais e cinza da casca de arroz

estudo, a influência da sílica sobre a vulcanização foi ainda mais pronunciada ${ }^{[18]}$;

b) Para os vulcanizados com cinza preta, os valores de $E_{a}$ são equivalentes aos das composições com negro de fumo. Provavelmente, a existência na composição química da cinza preta de dois componentes diferentes, sílica e carbono, seja o fator responsável pelos valores encontrados. Cotten ${ }^{[19]}$ sugere que os grupamentos funcionais contendo oxigênio, presentes na superfície do negro de fumo, exercem um profundo efeito adverso sobre a velocidade da reação de cura e o torque máximo, quando esta carga participa de formulações de borracha natural contendo o sistema CBS/enxofre;

c) Para as composições com cinza branca, a inesperada diminuição de $\mathrm{E}_{\mathrm{a}}$ com o aumento do teor de carga pode ser atribuída à presença de óxidos metálicos e outras impurezas na sua composição química, que levam a um efeito sinérgico com o $\mathrm{ZnO}$, como sugerido por Russel e colaboradores ${ }^{[20]}$.

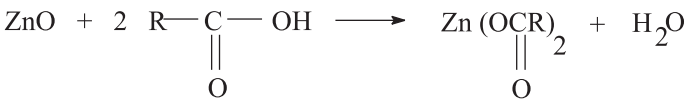<smiles></smiles><smiles>[R]OC(=O)OC(=O)O</smiles>

Figura 2. Reação entre os grupamentos silanóis, presentes na carga de sílica, e o estearato de zinco $^{[18]}$ 


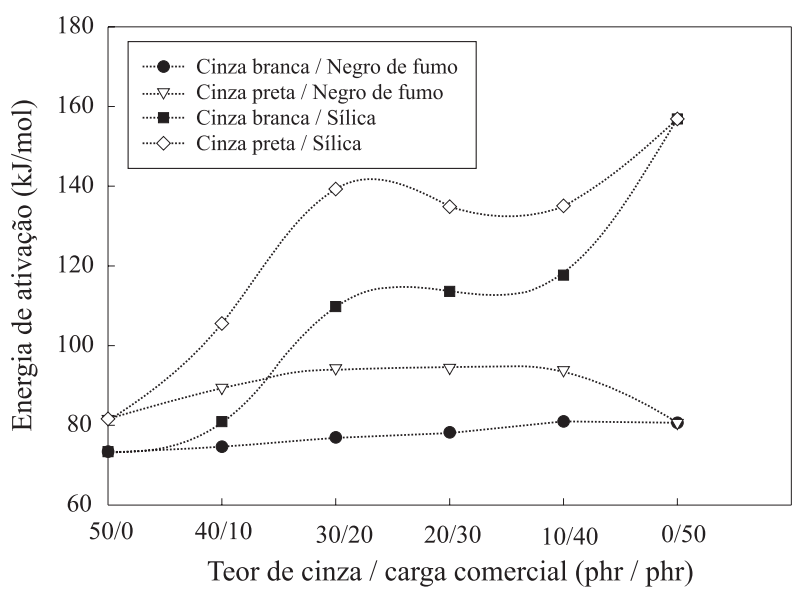

Figura 3. Variação da energia de ativação aparente para a vulcanização das composições de borracha natural - efeito da substituição parcial das cargas comerciais pela cinza da casca de arroz

Prosseguindo o estudo da cinética de vulcanização, as cargas comerciais foram substituídas parcialmente pelas cinzas preta e branca, em incrementos de $10 \mathrm{phr}(0 / 50,10 / 40,20 /$ $30,30 / 20,40 / 10,50 / 0)$. O procedimento experimental foi o mesmo descrito para as cargas adicionadas isoladamente $\mathrm{e}$ os valores de $\mathrm{E}_{\mathrm{a}}$ das diferentes composições, calculados pela equação 2, estão ilustrados na Figura 3.

A substituição parcial das cargas comerciais pelas cinzas $\mathrm{CB}$ e CP novamente vem confirmar o efeito catalítico da cinza branca (CB) sobre a cinética de vulcanização. Novamente, isto é resultado das diferenças entre as cinzas. A cinza preta (CP) pode ser considerada como uma carga extremamente porosa, em função da discrepância entre o tamanho de partícula e a área específica, conforme observado na Tabela 1. O método de adsorção de nitrogênio BET, usado para a obtenção da área específica, mede todas as áreas acessíveis à molécula de nitrogênio, como poros, trincas e cavidades, presentes nos agregados de carga. Como a molécula de nitrogênio é muito pequena, o método de adsorção de $\mathrm{N}_{2}$ pode resultar em áreas específicas muito maiores do que os valores reais. $\mathrm{Na}$ Tabela 1, a área específica para CP é $109 \mathrm{~m}^{2} / \mathrm{g}$, mas os poros contribuem com $80,1 \%$ desse valor.

É sabido que as partículas de negro de fumo com poros e trincas apresentam valores altos de áreas específicas, em comparação aos negros de fumo de tamanho de partícula semelhante, porém sem estas características. Isto pode resultar em um efeito retardante na cura, ocasionado pela adsorção e a conseqüente inativação de ingredientes da composição (aceleradores, por exemplo), que ficariam aprisionados nestas cavidades.

Para as composições de borracha natural com cinza branca, o efeito catalítico talvez seja resultado de características como menor área específica e ausência de poros, menor teor de carbono (perda por ignição). Embora exista um maior teor de sílica (97\%), a presença nesta carga de uma quantidade expressiva de óxidos metálicos (19,3\% maior do que na cinza preta) estaria fornecendo uma quantidade adicional de agentes de ativação. Russel et al. ${ }^{20}$ observaram que nenhum óxido sozinho é tão eficiente quanto o óxido de zinco, mas alguns óxidos $\left(\mathrm{MgO}, \mathrm{SnO}_{2}, \mathrm{~Pb}_{3} \mathrm{O}_{4}, \mathrm{Sb}_{2} \mathrm{O}_{3}, \mathrm{TeO}_{2}, \mathrm{Fe}_{2} \mathrm{O}_{3}\right.$, $\mathrm{Cr}_{2} \mathrm{O}_{3}$ ), quando usados em combinação com o de zinco, mostram um efeito positivo sobre a vulcanização.

\section{Conclusões}

A adição da cinza da casca de arroz a composições de borracha natural revelou diferentes efeitos sobre a cinética de vulcanização, em comparação com cargas convencionais como negro de fumo e sílica, dependendo do tipo de cinza usado.

Considerando que a reação de vulcanização segue uma cinética de primeira ordem, a presença de quantidades crescentes de cinza preta, mais porosa e com menor teor de sílica, levou a um comportamento muito semelhante ao do negro de fumo. Já a cinza branca apresentou um ligeiro efeito acelerador provocado pela adição de teores mais altos desta carga.

A substituição parcial das cargas comerciais pelas cinzas novamente mostrou um efeito catalítico mais pronunciado no caso da cinza $\mathrm{CB}$ e que pode ser atribuído à presença de pequenas quantidades de diferentes óxidos, os quais, em combinação com o óxido de zinco introduzido na formulação, produziriam um sinergismo em relação à velocidade de vulcanização.

\section{Agradecimentos}

Os autores agradecem à CAPES pelo suporte financeiro e à EMBRAPA pela doação da cinza.

\section{Referência Bibliográfica}

1. Costa, H. M.; Visconte, L. L. Y.; Nunes, R. C. R. \& Furtado, C. R. G. - J. Appl. Polym. Sci., 76, p.1019 (2000).

2. Costa, H. M.; Visconte, L. L. Y.; Nunes, R. C. R. \& Furtado, C. R. G. - J. Appl. Polym. Sci., 83, p.2331 (2002).

3. Nasir, M.; Poh, B. T. \& Nig, P. S. - Eur. Polym. J., 24, p.961 (1988).

4. Chough, S. H. \& Chang, D. H. - J. Appl.. Polym. Sci., 61, p.449 (1996).

5. Coran, A. Y. - Chemtech, february, p.106 (1983).

6. Morell, S. H. - "The chemistry and technology of vulcanization", in: Rubber Technology and Manufacture, cap.5, C. M. Blow, Newnes-Butterworths, London (1975).

7. Brabin, W. W. \& Rodgers, M. B. - "The Science of rubber compounding", in: The Science and Technology of Rubber, cap.9, F. R. Eirich \& B. Erman, Academic Press, New York (1994).

8. Coran, A. Y. - "Vulcanization”, in: The Science and Technology of Rubber, cap.7, F. R. Eirich \& B. Erman, Academic Press, New York (1994). 
9. Bateman, L.; Moore, C. G.; Porter, B. \& Saville, B. - "Vulcanization", in: The Chemistry and Physics of Rubber-Like Substances, cap.15, Bateman L. (ed.), Maclaren and Sons Ltd., London (1963).

10. Campbell, R. H. \& Wise, R. W. - Rubber Chem. Technol., 37, p.635 (1964).

11. Coran, A. Y. - Rubber Chem. Technol., 37, p.679 (1964).

12. Krejsa, M. R.; Koening, J. L. \& Sullivan, A. B. - Rubber Chem. Technol., 67, p.348 (1994).

13. Campbell, D. S. - J. Appl. Polym. Sci., 14, p.1409 (1970).

14. Parks, C. R.; Parker, D. K.; Chapman, D. A. \& Cox, W. L. - Rubber Chem. Technol., 43, p.572 (1970).
15. Morrison, N. J. \& Porter, M. - Rubber Chem. Technol., 57, p.63 (1984).

16. Parks, C. R.; Parker, D. K. \& Chapman, D. A. - Rubber Chem. Technol., 45, p.467 (1972).

17. Milligan, B. - Rubber Chem. Technol., 39, p.1115 (1966).

18. Wagner, M. P. - Rubber Chem. Technol., 49, p.703 (1976).

19. Cotten, G. R. - Rubber Chem Technol., 45, p.129 (1972).

20. Russel, R. M.; Skinner, T. D. \& Watson, A. A. - Rubber Chem. Technol., 41, p.418 (1968).

Recebido: 06/09/002 Aprovado: 12/02/03 